This is the final peer-reviewed accepted manuscript of:

Giovanna Calò, Gaetano Bellanca, Ali Emre Kaplan, Franco Fuschini, Marina Barbiroli, Michele Bozzetti, Paolo Bassi, and Vincenzo Petruzzelli. 2018. Integrated Vivaldi antennas, an enabling technology for optical wireless networks on chip. In Proceedings of the 3rd International Workshop on Advanced Interconnect Solutions and Technologies for Emerging Computing Systems (AISTECS '18). Association for Computing Machinery, New York, NY, USA, Article 1, 1-4.

The final published version is available online at https://doi.org/10.1145/3186608.3186609

Rights / License:

The terms and conditions for the reuse of this version of the manuscript are specified in the publishing policy. For all terms of use and more information see the publisher's website.

This item was downloaded from IRIS Università di Bologna (https://cris.unibo.it/)

When citing, please refer to the published version. 


\section{Integrated Vivaldi antennas, an enabling technology for optical wireless networks on chip}

\author{
Giovanna Calò \\ Dipartimento di Inegneria Elettrica e \\ dell'Informazione, Politecnico di Bari \\ Bari, Italy \\ giovanna.calo@poliba.it \\ Franco Fuschini \\ Department of Electrical, Electronic \\ and Information Engineering, \\ University of Bologna \\ Bologna, Italy \\ franco.fuschini@unibo.it
}

\author{
Gaetano Bellanca \\ Department of Engineering, \\ University of Ferrara \\ Ferrara, Italy \\ gaetano.ballanca@unife.it \\ Marina Barbiroli \\ Department of Electrical, Electronic \\ and Information Engineering, \\ University of Bologna \\ Bologna, Italy \\ marina.barbiroli@unibo.it
}

\author{
Ali Emre Kaplan \\ Department of Engineering, \\ University of Ferrara \\ Ferrara, Italy \\ aliemre.kaplan@unife.it
}

\author{
Michele Bozzetti \\ Dipartimento di Inegneria Elettrica e \\ dell'Informazione, Politecnico di Bari \\ Bari, Italy \\ michele.bozzetti@poliba.it
}

\author{
Paolo Bassi \\ Department of Electrical, Electronic \\ and Information Engineering, \\ University of Bologna \\ Bologna, Italy \\ paolo.bassi@unibo.it
}

\author{
Vincenzo Petruzzelli \\ Dipartimento di Inegneria Elettrica e \\ dell'Informazione, Politecnico di Bari \\ Bari, Italy \\ vincenzo.petruzzelli@poliba.it
}

\begin{abstract}
In this paper, we propose an integrated Vivaldi antenna, coupled to a silicon waveguide, for wireless Optical Network-on-Chip applications. On-chip wireless propagation characteristics and point-topoint link performances in homogeneous and multilayered medium are investigated and discussed as well. The proposed structure allows the implementation of wireless communication at optical frequencies and it can lead to a completely new approach in network design, allowing hybrid wireless/wired communications on the same die. This hybrid solution, in fact, can mitigate the problems related to the design and the fabrication of complex switching matrices in large networks, where long paths suffer of crosstalk and loss issues.
\end{abstract}

\section{CCS CONCEPTS}

- Hardware $\rightarrow$ Photonic and optical interconnect; Wireless devices; Radio frequency and wireless interconnect;

\section{KEYWORDS}

Optical antennas, Nanoantennas, Nanophotonics, Surface plasmons, Wireless optical communications, Optical networks on chip, Optical Wireless Networks on Chip

\section{INTRODUCTION}

Chip Multiprocessors are the state of the art solution to face the constant need of increasing computing system efficiency. They exploit parallel code execution using multiple threads across the cores. The efficient interconnection of these cores is of primary importance to avoid communication bottleneck, especially as the number of cores scales up. The requirements of high bandwidth, low-power and low-latency cannot be matched by traditional point-to-point connections through dedicated wires. Therefore, Network-on-chips (NoCs) have emerged as a technology enabling a high degree of integration in multi-core systems on chip. The NoCs reduce the wiring complexity and increase communication efficiency. However, owing to limitations on data bandwidth and increasing power consumption determined by metal interconnections, important performance issues arise from this approach.

The recent developments in silicon photonics have enabled promising alternatives that, through photonic components, allow the data transmission at hundreds of $\mathrm{Gb} / \mathrm{s}$ data rates over an Optical Network-on-Chip (ONoC) layer [3, 6, 12, 14]. The proposed ONoC-based architectures rely on an optical layer, stacked with electronic layers, that allow the communications between different cores in the optical domain, through complex networks with latencies in the order of tens of ns. Major drawbacks of the guided wave solutions are layout and routing algorithms complexity [7, 17]. 
An alternative emerging technology to overcome performance limitations of traditional NoCs is the Wireless Network-on-Chip (WiNoC), which exploits on-chip wireless communication to perform hybrid wired/wireless links. Silicon integrated antennas, operating in the millimeter-wave range, replace wired channels so that transmission performance, power consumption and long distant communication problems of traditional wired NoCs can be addressed simultaneously, reaching tens of $\mathrm{Gb} / \mathrm{s}$ data rates and reducing the traditional NoC latencies of $20-45 \%$ [8]. However, since their size is in the order of a few millimeters, these antennas suffer for low integrability and are not always a viable solution to replace ONoCs at the CMP communication level.

A completely novel approach which can combine the advantages of both the $\mathrm{ONoC}$ and the $\mathrm{WiNoC}$ is the on-chip wireless connection through optical antennas. The on-chip integration of miniaturized optical radiating elements, such as nanoantennas, could enable optical wireless communication also at the "small world" scale of the CMP. Nanoantennas have been recently proposed to enhance the efficiency of photodetection, sensing, heat transfer, and spectroscopy $[15,16]$.

An emerging research trend regards the demonstration of nanoantennas for optical communication purposes [1]. Recent researches have investigated different plasmonic antennas for on-chip wireless communication such as dipole and horn antennas [11, 18, 20], but the proposed solutions are based on all-plasmonic structures, which do not address the problem of integrating such devices within SOIbased optical networks on chip. An interesting solution based on dielectric antennas has also been recently published [9], and on chip optical wireless communication in the millimeter range has been demonstrated.

In this paper, we propose plasmonic Vivaldi antennas coupled to silicon ( $\mathrm{Si}$ ) waveguides as an enabling technology for OWiNoCs. The advantage of using antennas integrated with Si waveguides is the compatibility with SOI technologies and components for the optical signal processing, such as modulators, filters, etc.. Therefore, the same signals propagating on optical waveguides can be directly used for wireless optical connections, thus increasing the network transparency. Here, we also report the simulation results of a wireless point-to-point link in an homogeneous and a layered medium, and discuss the effects of dielectric discontinuities on link performance.

\section{OPTICAL WIRELESS LINK BETWEEN INTEGRATED VIVALDI ANTENNAS}

Fig.1 (a) shows the conceptual scheme of the OWiNoC where hybrid wireless/wired connections are allowed by the full compatibility of the proposed Vivaldi antennas with SOI optical waveguides. Adjacent processors (or processor clusters) are connected through optical waveguides, whereas long-range communications are provided by optical highly-directive point-to-point wireless links. As schematized in Fig.1 (b), an input WDM signal, coming from a silicon integrated waveguide, is coupled to the nanoantenna and transmitted in the surrounding medium. After propagation, the signal is received by a second antenna and then coupled again to an optical waveguide. This approach based on all-optical signals allows bitrate transparency, ultra-high modulation rates, low latency and

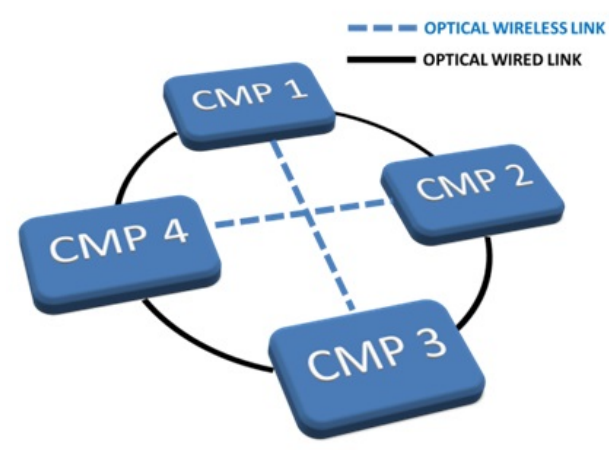

(a)

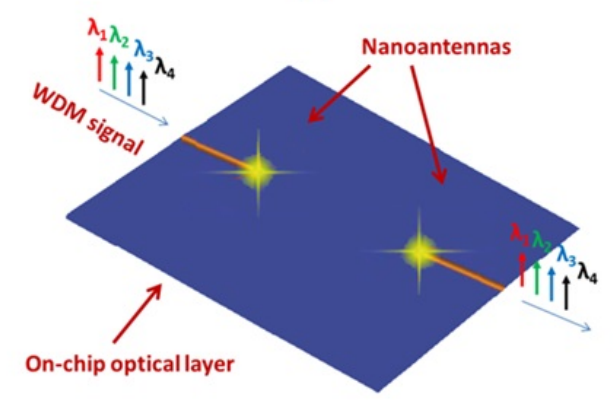

(b)

Figure 1: Schematic of (a) an hybrid wireless/wired optical connection between four processors, and of (b) an optical WDM wireless link integrated on the on-chip optical layer.

high bandwidth density. In fact, in this configuration, the same optical signals can propagate without requiring any conversion through either the dielectric waveguides or the wireless interconnections. Fig.2 (a) shows the schematic of the proposed Vivaldi antenna configuration. The optical signal is launched into a Silicon waveguide and it is vertically coupled to a plasmonic slotted waveguide, made of silver. After this hybrid Si-plasmonic coupler, the plasmonic slotted waveguide opens up into a Vivaldi antenna and radiates the signal in the surrounding medium, i.e. $\mathrm{SiO}_{2}$. The relevant geometrical parameters of the coupler, whose cross-section is schematized in Fig. 2 (b), were designed by applying the coupled mode theory (CMT) and the normal mode analysis [4, 19]. The design was validated and optimized by three-dimensional Finite Element Method (FEM) simulations [5]. The optimal geometrical parameters are summarized in Tab.1. After designing the coupling section, the Vivaldi antenna width is constrained by the slotted waveguide width, whereas the antenna behaviour can be optimized by varing the antenna length $L_{a}$. The optimization was performed by FDTD simulations [10] and the optimal antenna length $L_{a}=1.75 \mu \mathrm{m}$ was chosen, corresponding to the maximum antenna gain $G=9.9 \mathrm{~dB}$. Further details can be found in [2].

In addition to the optimal design of the radiating elements, a topic that needs careful evealuation is the electromagnetic propagation in an on-chip environment [13]. Fig.2 (c) shows a scheme of a wireless 
link between two Vivaldi antennas separated by a distance $d$. The two antennas are coupled to two Si waveguides and the multilayer environment typical of SOI-based optical devices is considered, i.e. a three layered environment, with stacked $\mathrm{Si}, \mathrm{SiO}_{2}$, and air layers. The scenario shown in Fig.2 (c) was analyzed by three-dimensional FDTD simulations [10] and can be considered as a starting point for understanding the effects of dielectric discontinuities on wireless propagation at optical wavelengths. Fig.3 (a) shows the electric field intensity (in logarithmic scale and in the $Y Z$ vertical plane) obtained for a wireless link in the multi-layered environment, with a distance $d=50 \mu \mathrm{m}$ between the two antennas. We used this link distance to allow full wave FDTD simulations of this scenario in a reasonable time. To simulate wireless propagation for longer distances other approaches such as, for example, ray-tracing, should be used.

For the sake of comparison, Fig.3 (b) shows the electric field intensity (in logarithmic scale and in the $Y Z$ vertical plane) obtained for the same wireless link but in an homogeneous medium, i.e. $\mathrm{SiO}_{2}$. As we can infer by comparing Figs.3 (a) and (b), in the multi-layered scenario, the propagation conditions strongly differ from the ones in homogeneous environment, where only the field spreading effect typical of free-space propagation is visible. As shown in Fig.3 (a), the presence of multiple layers introduces a waveguiding phenomenon which strongly influence the propagation. Fig.3 (c) shows the received power at the output waveguide of the receiving antenna, as a function of the wavelength, for the links shown in Figs. 3 (a) and (b). The received power has been obtained by computing the power flux at the output section for the fundamental mode of the silicon waveguide. The input power is $P_{i n}=0 \mathrm{dBm}$ and, therefore, the power $P_{r}$ calculated at the output port is directly the power budget of the connection. The overall received power at the wavelength $\lambda=1.55 \mu \mathrm{m}$ is $P_{r}=-36.29 \mathrm{dBm}$ in the case of homogeneous environment, whereas it becomes $P_{r}=-25.98 \mathrm{dBm}$ in the multilayer environment, with an increase of $10.31 \mathrm{~dB}$. This phenomenon is due to multiple reflections at the interfaces, that tend to confine the electromagnetic field in the central $\mathrm{SiO}_{2}$ layer, and reduce the power loss due to diffraction-induced free space attenuation. Another approach to cope with this problem is to use antenna arrays. With this solution, beam-steering would also be possible, thus enabling the possibility to include dynamic switching between links by redirecting the communication toward different destinations. Although free-space attenuation at optical wavelengths is high for distances compatible with the dimension of multiprocessor chip, it has been demonstrated that on-chip optical links are possible [9] with antennas having gains of the order of $20 \mathrm{~dB}$, which can be obtained by configuring arrays of integrated Vivaldi radiators.

\section{CONCLUSIONS}

In this paper we propose an integrated Vivaldi antenna for links in hybrid wireless/wired optical networks on chip. The Vivaldi antenna is coupled to a silicon waveguides through hybrid Siplasmonic coupler. This configuration guarantees a full compatibility with SOI technology and with optical components generally used for optical signal processing. The results of 3D-FDTD simulations of a point-to-point $50 \mu \mathrm{m}$ link in multilayered environment have been reported. The comparison between the propagation in

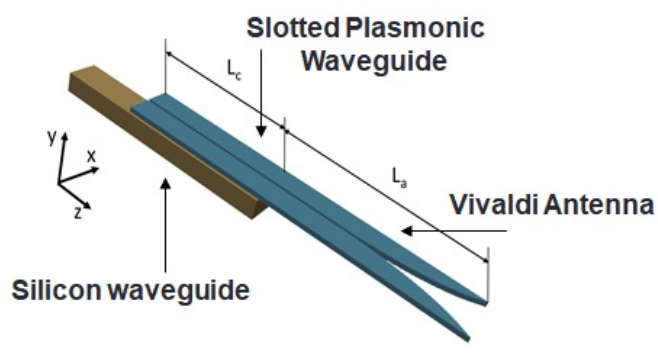

(a)

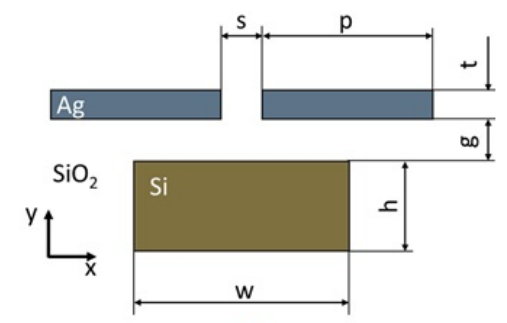

(b)

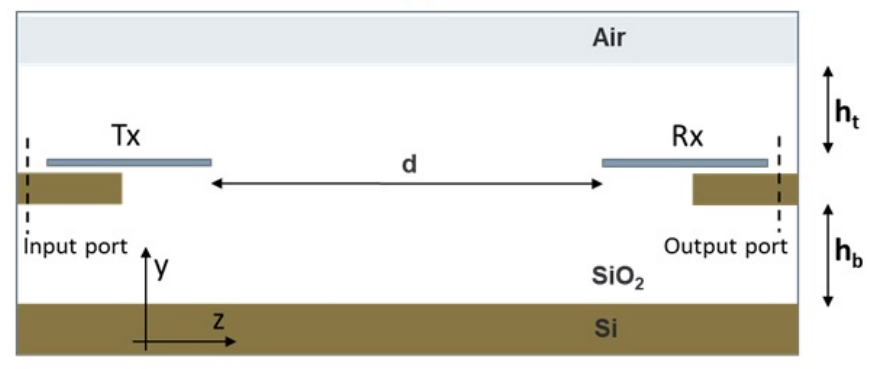

(c)

Figure 2: Schematic of (a) the integrated Vivaldi antenna coupled to a silicon waveguide, of (b) cross-section of the hybrid Si-plasmonic coupler, and of (c) the on-chip wireless link between two integrated Vivaldi antennas.

Table 1: Geometrical parameters of the cross-section of the Si-hybrid plasmonic coupler

\begin{tabular}{cc}
\hline Geometrical parameter & dimension [nm] \\
\hline gap between the Si and the Ag waveguides g & 80 \\
width of Si waveguide w & 380 \\
height of Si waveguide $\mathrm{h}$ & 220 \\
plasmonic waveguide width $\mathrm{p}$ & 270 \\
slot width s & 30 \\
thickness of the Ag layer t & 50 \\
length of the hybrid Si-plasmonic coupler $L_{c}$ & 1630 \\
\hline
\end{tabular}

homogeneous and in multi-layered environment demonstrates that the technology can strongly influence the link properties and cannot be ignored when designing the chip. In fact, the double interface in the multi-layer scenario induces an increase of the received power 


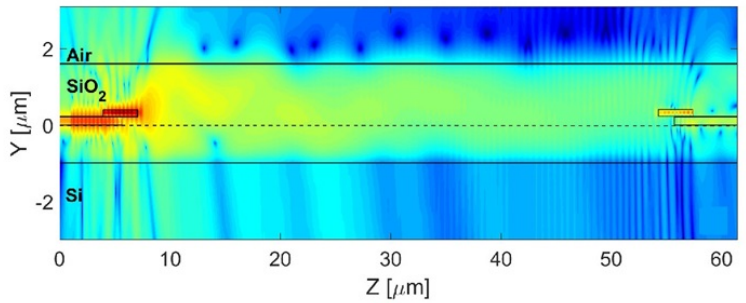

(a)

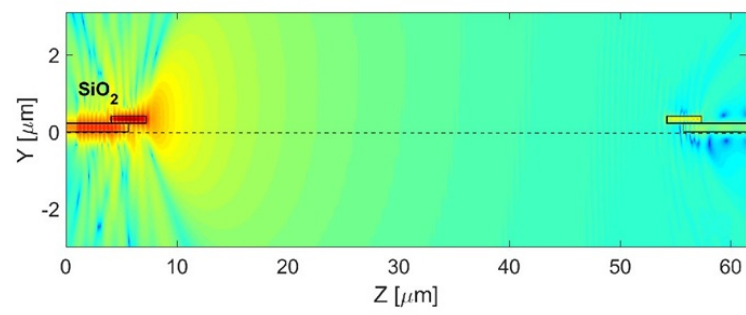

(b)

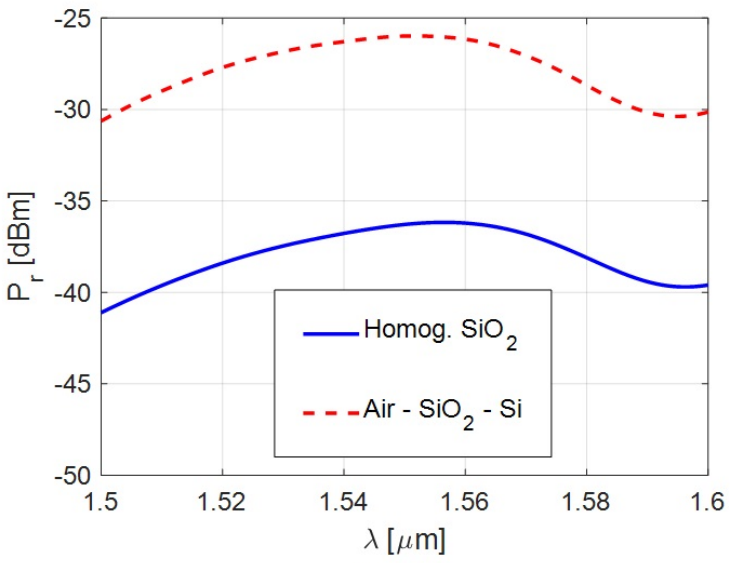

(c)

Figure 3: Electric field intensity (in logarithmic scale) obtained for wireless link in the multi-layered environment (a) and in the homogeneous $\mathrm{SiO}_{2}$ medium (b). (c) Received power $P_{r}$ (in $\mathrm{dBm}$ ) at the output section of the receiving antenna as a function of wavelength and for the two different investigated scenarios. The input power $\mathbf{P}_{t}$ is $0 \mathrm{dBm}$ and the wavelength is $\lambda=1.55 \mu \mathrm{m}$.

of about $10 \mathrm{~dB}$ with respect to the propagation in an homogeneous medium. The proposed results can be a first step toward the design of new network solutions and they can also be the basis for the development of propagation models and ray-tracing algorithms. We aim at developing an integrated approach that, by using ray-tracing, would allow to investigate optical propagation in on-chip realistic scenarios. This approach will also allow to take into account the presence of multiple interfering links, in order to evaluate the communication performance of the network in term, for example, of power needed for each link and BER.

\section{ACKNOWLEDGMENTS}

This research was supported by MIUR through the PRIN 2015 "Wireless Networks through on-chip Optical Technology - WiNOT" Project and by Apulia region project "Regional laboratory for synthesis and characterization of new organic and nanostructured materials for electronics, photonics, and advanced technologies". Gaetano Bellanca acknowledges support from "Bando per l'acquisizione di strumenti per la ricerca di Ateneo - Anno 2015" of the University of Ferrara.

\section{REFERENCES}

[1] A. Alú and N. Engheta. 2010. Wireless at the nanoscale: Optical interconnects using matched nanoantennas. Physical Review Letters 104, 21 (2010). https: //doi.org/10.1103/PhysRevLett.104.213902

[2] G. Bellanca, G. Caló, A.E. Kaplan, P. Bassi, and V. Petruzzelli. 2017. Integrated Vivaldi plasmonic antenna for wireless on-chip optical communications. Optics Express 25, 14 (2017), 16214-16227.

[3] A. Biberman and K. Bergman. 2012. Optical interconnection networks for highperformance computing systems. Reports on Progress in Physics 75, 4 (2012).

[4] G. Caló, A. D’Orazio, and V. Petruzzelli. 2012. Broadband mach-zehnder switch for photonic networks on chip. Fournal of Lightwave Technology 30, 7 (2012), 944-952.

[5] COMSOL Multiphysics 2017. (2017). http://www.comsol.com/

[6] S. Faralli, F. Gambini, P. Pintus, O. Liboiron-Ladouceur, Y. Xiong, P. Castoldi, F. Di Pasquale, N. Andriolli, and I. Cerutti. 2016. Bidirectional Transmission in an Optical Network on Chip with Bus and Ring Topologies. IEEE Photonics fournal 8, 1 (2016). https://doi.org/10.1109/JPHOT.2016.2526607

[7] F. Gambini, S. Faralli, P. Pintus, N. Andriolli, and I. Cerutti. 2015. BER evaluation of a low-crosstalk silicon integrated multi-microring network-on-chip. Optics Express 23, 13 (2015), 17169-17178.

[8] A. Ganguly, K. Chang, S. Deb, P.P. Pande, B. Belzer, and C. Teuscher. 2011. Scalable hybrid wireless network-on-chip architectures for multicore systems. IEEE Trans. Comput. 60, 10 (2011), 1485-1502.

[9] C. García-Meca, S. Lechago, A. Brimont, A. Griol, S. Mas, L. Sánchez, L. Bellieres, N.S. Losilla, and J. Martí. 2017. On-chip wireless silicon photonics: from reconfigurable interconnects to lab-on-chip devices. Light: Science \& Applications 6 (2017).

[10] Lumerical Solutions, Inc. 2017. (2017). http://www.lumerical.com/tcad-products/ fdtd/

[11] J.M. Merlo, N.T. Nesbitt, Y.M. Calm, A.H. Rose, L. D’Imperio, C. Yang, J.R. Naughton, M.J. Burns, K. Kempa, and M.J. Naughton. 2016. Wireless communication system via nanoscale plasmonic antennas. Scientific Reports 6 (2016). https://doi.org/10.1038/srep31710

[12] D.A.B. Miller. 2009. Device requirements for optical interconnects to silicon chips. Proc. IEEE 97, 7 (2009), 1166-1185.

[13] M. Nafari, L. Feng, and J.M. Jornet. 2017. On-chip wireless optical channel modeling for massive multi-core computing architectures. IEEE Wireless Communications and Networking Conference, WCNC (2017). https://doi.org/10.1109/ WCNC.2017.7925962

[14] M. Nikdast, G. Nicolescu, S. Le Beux, and J. Xu. 2017. Photonic Interconnects for Computing Systems. River Publishers.

[15] L. Novotny. 2011. From near-field optics to optical antennas. Physics Today 64, 7 (2011), 47-52.

[16] L. Novotny and N. Van Hulst. 2011. Antennas for light. Nature Photonics 5, 2 (2011), 83-90.

[17] M. Ortin-Obon, M. Tala, L. Ramini, V. Vinals-Yufera, and D. Bertozzi. 2017. Contrasting Laser Power Requirements of Wavelength-Routed Optical NoC Topologies Subject to the Floorplanning, Placement, and Routing Constraints of a 3-D-Stacked System. IEEE Transactions on Very Large Scale Integration (VLSI) Systems 25, 7 (2017), 2081-2094

[18] Y. Yang, Q. Li, and M. Qiu. 2016. Broadband nanophotonic wireless links and networks using on-chip integrated plasmonic antennas. Scientific Reports 6 (2016). https://doi.org/10.1038/srep19490

[19] A. Yariv. 1973. Coupled-Mode theory for guided-wave optics. IEEE fournal of Quantum Electronics 9, 9 (1973), 919-933.

[20] L. Yousefi and A.C. Foster. 2012. Waveguide-fed optical hybrid plasmonic patch nano-antenna. Optics Express 20, 16 (2012), 18326-18335. 